\title{
Metastatic Clear Cell Renal Cell Carcinoma with an Unknown Primary in a Living Donor Kidney Transplant Recipient
}

\author{
Ahmed Elfadaly*, Nelish Ardeshna, Obinna Ekwenna and Jorge Ortiz \\ Department of Surgery, University of Toledo Medical Center, 3000 Arlington Avenue, Toledo, OH 43614, USA
}

\begin{abstract}
Background: Renal cell carcinoma (RCC) commonly metastasizes, and unusual sites of spread are characteristic. However, metastatic RCC without an identified primary site is extremely rare, and only a few cases have been documented. We report the first case of clear cell RCC identified in retroperitoneal, supraclavicular, and mediastinal lymph nodes (LN) without a primary site in a kidney transplant patient.

Case report: A 68-year-old patient presented with abdominal pain accompanied by weight loss, decreased appetite, and night sweats 6 months post female living donor kidney transplant. A CT scan revealed retroperitoneal, supraclavicular, and mediastinal lymphadenopathy. An excisional supraclavicular LN biopsy stained for markers consistent with RCC (pancytokertain, PAX8, CD10, and vimentin). Subsequent karyotyping revealed a female aneuploid cell line and raised suspicion for a donor derived RCC. A primary site for the RCC could not be identified on imaging of the native and allograft kidneys. The patient expired waiting for the pathology report, and an autopsy was performed to identify the origin of the RCC. Autopsy could not identify a primary site and cytologic studies indicated that the cell line was derived from the patient. Additional chromosomal abnormalities identified in the cytologic studies (loss of VHL, PBRM1, SETD2, BAP1, CDKN2A/B TP53) narrowed the diagnosis to clear cell RCC.
\end{abstract}

Conclusion: Metastatic RCC can present without a primary site. IHC plays a vital role in making the diagnosis and cytologic studies can confirm the diagnosis and exclude the renal allograft as the origin in a kidney transplant patient.

\section{Introduction}

Worldwide, renal cell carcinoma (RCC) is the ninth most common cancer and has an annual incidence of approximately 300,000. At the time of diagnosis, 25 to $30 \%$ of patients with RCC have metastases [14]. The metastases can involve any organ, and unusual sites of spread are considered characteristic. Metastatic RCC without a primary site is rare and only a few cases have been documented.

Renal transplant is the treatment of choice in end-stage renal disease. Immunosuppression is required to improve graft survival, but it can increase the risk of RCC. We present a case of clear cell RCC with retroperitoneal, supraclavicular, and mediastinal lymph node metastases without a primary site in a 68-year-old status post living donor renal transplant patient.

\section{Case Presentation}

A 68-year-old male presented to the emergency department with severe epigastric pain six months post living donor renal transplant from his daughter-in-law. The patient had an uncomplicated transplant course and was in his usual state of health until the onset of fatigue and constant abdominal pain one week prior. He also reported weight loss, decreased appetite, and night sweats. Past medical history included hypertension, diabetes type II, basal cell carcinoma, and coronary artery bypass surgery. The physical exam was unremarkable except for jaundice and pallor. An abdominal CT scan, colonoscopy, and esophagogastroduodenoscopy were performed several days earlier at a different facility and did not show acute pathology. He was admitted for further workup due to the severity of the abdominal pain.
A repeat CT scan revealed retroperitoneal, supraclavicular, and mediastinal lymphadenopathy. Post-transplant lymphoproliferative disorder (PTLD) was suspected, and a retroperitoneal fine needle biopsy was performed. The biopsy revealed vacuolated malignant cells with nuclear enlargement, hyperchromasia with prominent nucleoli, and easily identifiable mitotic figures (Figures 1-3). The tissue was stained for a battery of markers (Table 1). Instead of a lymphoma, both the immunohistochemistry (IHC) and morphology results suggested a poorly differentiated carcinoma inconsistent with a primary site.

A PET scan was performed while the results for the retroperitoneal core biopsy were pending. It showed PET-avid adenopathy beginning at level of the thyroid cartilage and the left side of the neck extending inferiorly into the mediastinum. There was additional adenopathy in the retrocrural area bilaterally and in the peritoneum left of the aorta. The PET scan results and the patient's clinical picture of weight loss, decreased appetite, and night sweats were suggestive of a high-grade B-cell lymphoma. An excisional supraclavicular lymph node biopsy was performed because of the conflicting findings.

${ }^{\star}$ Correspondence to: Ahmed Elfadaly, MD, Department of Surgery, University of Toledo Medical Center, 3000 Arlington Avenue, Toledo, OH 43614, Tel: 419277-6529; E-mail: Ahmed.Elfadaly@utoledo.edu

Key words: imetastasis; renal cell carcinoma; unknown primary

Received: May 12, 2018; Accepted: May 20, 2018; Published: May 28, 2018 


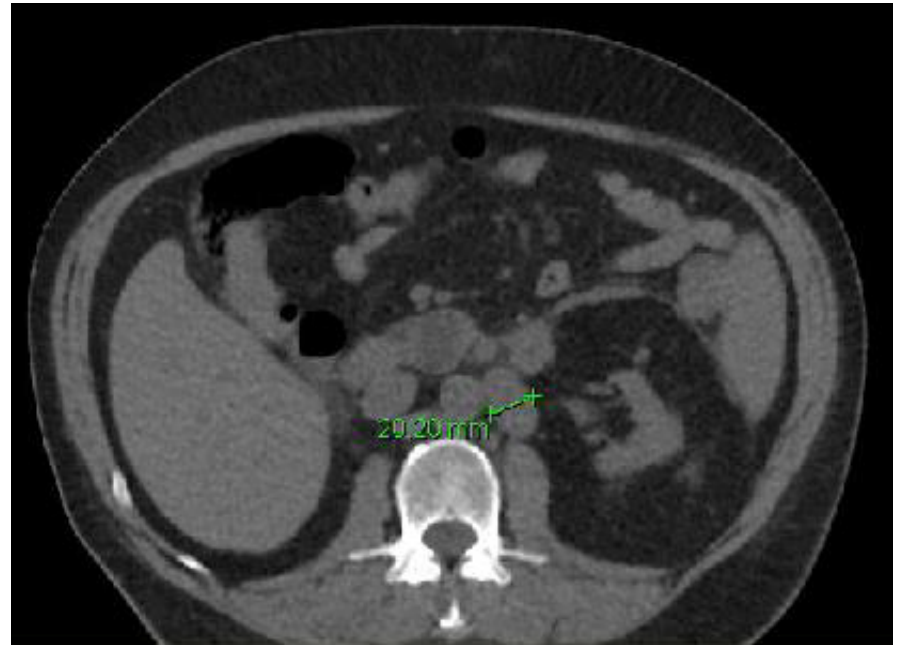

Figure 1. Ct abdomen without contrast showing enlargement of the left para-aortic LN

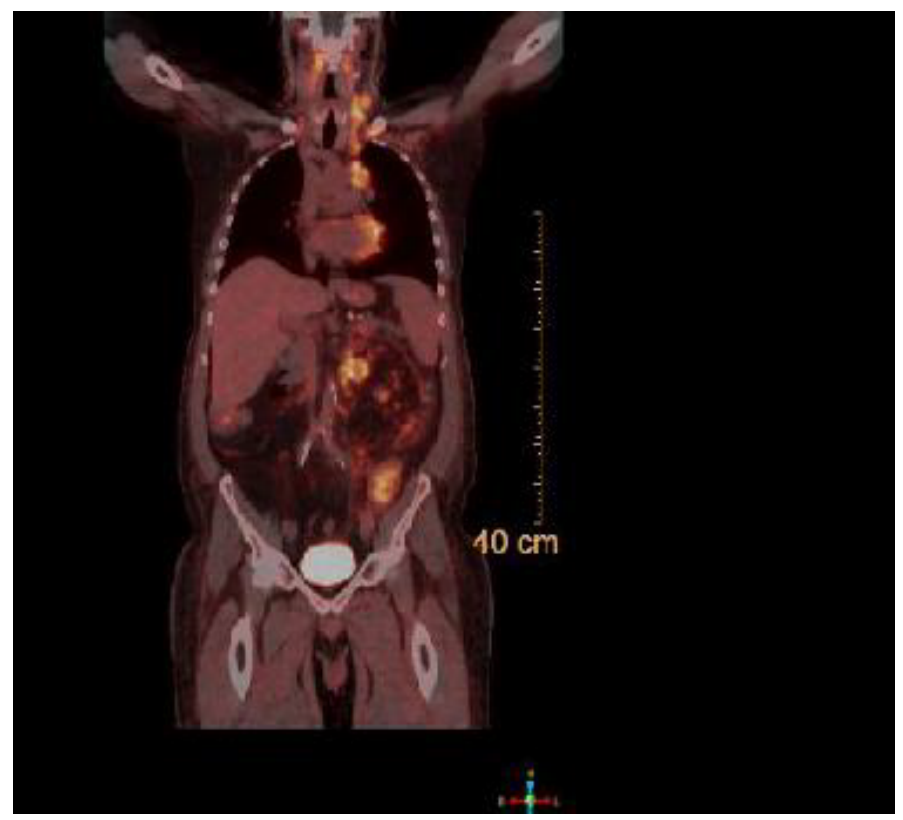

Figure 2. Whole body PET CT showing avid uptake in the left supraclavicular LN, mediastinal LN and para-aortic LN

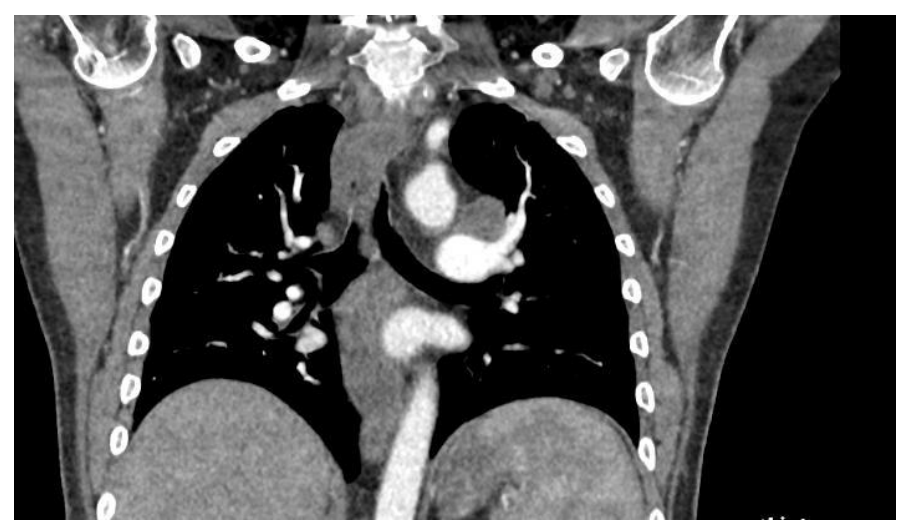

Figure 3. Ct scan of chest with IV contrast, coronal section showing enlarged mediastinal LN
Flow cytometry of the biopsy was negative for B-cell monoclonality and T-cell aberrancy, ruling out a high-grade B-cell lymphoma. Microscopy was consistent with RCC and showed highly anaplastic cells with some nesting but no definite gland formation. The biopsy stained positive for markers most consistent with RCC (Table 1). An abdominal CT scan was suspicious but not conclusive for a primary site on both kidneys. Pre-donation and pre-transplant CT scans were reviewed, but no specific lesions were identified. The patient was diagnosed with metastatic high-grade RCC with an unknown primary site.

To determine whether the metastatic RCC foci were donor derived (XX) or recipient derived (XY), karyotyping was performed. Analysis identified one normal male diploid cell line and an aneuploid female cell line. The remaining cultured cells were used for a comparative genomic hybridization and single nucleotide polymorphism (CGH/ SNP) studies to identify the tumor cell origin. However, there were insufficient tumor cells present.

The patient's health declined rapidly and he expired while waiting for the pathology report, so tumor cells for the CGH/SNP studies could not be harvested. A limited post-mortem that included examination of

Table 1. IHC Profile of Retroperitoneal Core Biopsy and Supraclavicular Excisional Biopsy

\begin{tabular}{|c|c|c|}
\hline Antibody Stain & $\begin{array}{c}\text { Retroperitoneal core } \\
\text { biopsy }\end{array}$ & $\begin{array}{c}\text { Supraclavicular excisional } \\
\text { biopsy }\end{array}$ \\
\hline \multicolumn{3}{|c|}{ Typical Carcinoma Markers } \\
\hline Pan-keratin & + & + \\
\hline Oscar Keratin & + & + \\
\hline EMA & - & + \\
\hline \multicolumn{3}{|c|}{ Typical Renal Markers } \\
\hline PAX8 & + & + \\
\hline CD10 & & + \\
\hline Vimentin & & + \\
\hline \multicolumn{3}{|c|}{ Other Markers } \\
\hline ALK & & - \\
\hline Calretinin & & - \\
\hline CD3 & - & - \\
\hline $\mathrm{CD} 20$ & - & - \\
\hline CD30 & - & - \\
\hline CD31 & - & + \\
\hline CD34 & & + \\
\hline CD45 & & - \\
\hline $\mathrm{CDX} 2$ & - & 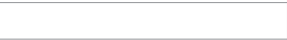 \\
\hline CK2 & - & \\
\hline CK7 & - & - \\
\hline CK10 & & - \\
\hline Desmin & - & \\
\hline GATA3 & - & \\
\hline Hep-Par1 & & - \\
\hline FL-1 & - & \\
\hline Mart1 & & - \\
\hline $\mathrm{p} 40$ & - & \\
\hline PAX5 & - & \\
\hline PLAP & & - \\
\hline PSA & & - \\
\hline PSAP & & - \\
\hline Synaptophysin & & - \\
\hline S100 & & - \\
\hline S1100 & - & \\
\hline TTF1 & - & - \\
\hline
\end{tabular}


both native and donor kidneys was performed to clarify the origin of the tumor cells and determine the primary site.

The left native kidney showed a benign leiomyoma with cortical atrophy, and the right native kidney showed a simple renal cyst with cortical atrophy. The renal allograft had transplant associated glomerulopathy. Microscopic examination revealed that both the native kidneys had glomerular sclerosis, KW lesions, and thyroidization consistent with diabetic nephropathy and ESRD. A malignant tumor in the renal parenchyma of the native or allograft kidney was not identified.

The tissue samples from the right native kidney, allograft kidney, and supraclavicular lymph node were sent for cytologic examination. The native kidney matched 11 of 11 informative markers of the supraclavicular lymph node, while only matching 1 of 11 markers of the allograft kidney. This indicated that the tumor originated from the patient's cell line. Additionally, chromosomal microarray analysis revealed a complex XY karyotype that was consistent with a duplicated $\mathrm{X}$ chromosome and a mosaic loss of the $\mathrm{Y}$ chromosome. This further confirmed that the tumor origin was from the patient. Additional tumor mutation screening of the supraclavicular lymph node identified chromosomal abnormalities including loss of chromosome $1 \mathrm{p}$ (including ARID1A), chromosome 3 (including VHL, PBRM1, SETD2, BAP1), chromosome 9 with concurrent loss of 9p21.3 (resulting in homozygous deletion of CDKN2A/B) and chromosome 17p13.2q21.3 (including TP53). These results were consistent with clear cell RCC with a cytogenic match to the native tissue.

\section{Discussion}

Kidney transplant patients are prone to malignancy because they have a history of renal disease and are immunosuppressed. Cancer has a 10 -year incidence rate of $20 \%$ in this population [5]. PTLD is the second most common type of malignancy in solid organ transplants [6]. It was suspected initially because PTLD frequently presents as lymphadenopathy and can cause abdominal pain. However, microscopy and IHC revealed a poorly differentiated neoplasm most consistent with high-grade RCC instead.

The next step was to determine the origin of the RCC. The native kidneys of transplant patients can develop RCC at a rate that is 10 to 100 times the general population [7]. Although less prevalent, RCC can also develop in the allograft kidney as a result of de novo mutations or transmission of occult malignant cells. It is important to identify the origin of the malignant cells because the donor may have residual tumor present or be at risk for a high-grade neoplasm. Initial karyotyping results revealed the presence of an aneuploidy female cell line. This finding made further studies necessary because errors in replication and loss of chromosomal stability in malignant cells can result in a female cell line [8]. Cytological examination of supraclavicular lymph node post autopsy determined that the female cell line resulted from a duplicated X chromosome and a mosaic loss of the Y chromosome. The results clarified that the cell line came from the patient, and the donor was determined to not be the origin of the cancer.

The diagnosis of metastatic RCC is not straightforward due to the morphological difference between the metastatic foci and the primary origin site. IHC is often used to assist in the diagnosis. It is not entirely specific in poorly differentiated neoplasms, but a diagnosis may be suggested by the markers present. The initial retroperitoneal core biopsy was positive for Pan-kertain (OSCAR) and EMA, while negative for CD45, PLAP, and synaptophysin (Table 1). This IHC profile suggests a carcinoma [9]. A lymphoma was ruled out because the neoplasm was negative for CD45 [10]. Furthermore, the supraclavicular lymph node excisional biopsy was positive for Pan-keratin, Pax8, CD10, and vimentin, which is most consistent with RCC [11]. Although there was suspicion, a primary renal site was not conclusively located on CT scans.

After the patient's death, a limited autopsy and cytogenic studies were performed that confirmed the diagnosis of metastatic RCC with no primary. The pathologist could not locate a tumor in the renal parenchyma of the native or allograft kidneys. A chromosomal microarray analysis revealed the loss of VHL, PBRM1, SETD2, BAP1, $\mathrm{CDKN} 2 \mathrm{~A} / \mathrm{B}$, and TP53 genes, and narrowed the diagnosis of RCC to the subtype of clear cell carcinoma. Mutations in these genes are commonly implicated in clear cell RCC [12].

Metastatic RCC without a primary is extremely rare and has been reported in a small number of case studies [2,3]. All cases were identified by IHC staining, tumor sequencing, and a few with molecular genetic analyses. Potential reasons for finding no primary site can be that the lesion remains occult, involutes or metastatic RCC show less differentiated pattern [2]. Experts have identified differences in molecular mechanisms between RCC and their metastases, which can make the metastases more aggressive than the primary cancer [13]. One study provided evidence that early and late metastases of RCC show an increasingly varied genetic expression profile as the time interval widens [14]. The altered profile of the metastases may result in a metastatic lesion prior to the primary. Regardless of the mechanism, IHC plays a key role in identifying RCC at a metastatic site when there is no primary renal site identified.

\section{Conclusion}

Metastatic RCC without a primary site involvement is rare. We report a unique case of a patient with a history of female living donor kidney transplant who developed metastatic RCC without a primary renal site. Additionally, this case represents the value of IHC and karyotyping in supporting the correct diagnosis.

Funding: This study was not funded.

\section{Compliance with Ethical Standards:}

Conflict of Interest: On behalf of all authors, the corresponding author states that there is no conflict of interest.

Informed consent: Informed consent was obtained from all individual participants included in the study.

Research involving human participants: All procedures performed in studies involving human participants were in accordance with the ethical standards of the institutional and/or national research committee and with the 1964 Helsinki declaration and its later amendments or comparable ethical standards.

Ethical Approval: For this type of study formal consent is not required.

\section{References}

1. Motzer RJ, Bander NH, Nanus DM (1996) Renal-cell carcinoma. $N$ Engl J Med 335 865-875. [Crossref]

2. Bhatia S, Ng S, Hodder SC (2010) Metastatic cutaneous head and neck renal cell carcinoma with no known primary: case report. Br J Oral Maxillofac Surg 48: 214 215. [Crossref]

3. Wayne M, Wang W, Bratcher J, Cumani B, Kasmin F, et al. (2010) Renal cell cancer without a renal primary. World J Surg Oncol 8: 18. [Crossref] 
4. Jonasch E, Gao J, Rathmell WK (2014) Renal cell carcinoma. BMJ 349: g4797. [Crossref]

5. Zhang J, Ma L, Xie Z, Guo Y, Sun W, et al. (2014) Epidemiology of post-transplant malignancy in Chinese renal transplant recipients: a single-center experience and literature review. Med Oncol 31: 32. [Crossref]

6. Zafar SY, Howell DN, Gockerman JP (2008) Malignancy after solid organ transplantation: an overview. Oncologist 13: 769-778. [Crossref]

7. Leveridge M, Musquera M, Evans A, Cardella C, Pei Y, et al. (2011) Renal cell carcinoma in the native and allograft kidneys of renal transplant recipients. J Urol 186: 219-223. [Crossref]

8. Arseneault M, Monlong J, Vasudev NS, Laskar RS, Safisamghabadi M, et al. (2017) Loss of chromosome Y leads to down regulation of KDM5D and KDM6C epigenetic modifiers in clear cell renal cell carcinoma. Sci Rep 7: 44876. [Crossref]
9. Oien KA, Dennis JL (2012) Diagnostic work-up of carcinoma of unknown primary: from immunohistochemistry to molecular profiling. Ann Oncol 23: x271-277. [Crossref]

10. Caldwell CW, Patterson WP (1991) Relationship between CD45 antigen expression and putative stages of differentiation in B-cell malignancies. Am J Hematol 36: 111115. [Crossref]

11. Truong LD, Shen SS (2011) Immunohistochemical diagnosis of renal neoplasms. Arch Pathol Lab Med 135: 92-109. [Crossref]

12. Cancer Genome Atlas Research N (2013) Comprehensive molecular characterization of clear cell renal cell carcinoma. Nature 499:43-49. [Crossref]

13. Semeniuk-Wojtas A, Stec R, Szczylik C (2016) Are primary renal cell carcinoma and metastases of renal cell carcinoma the same cancer? Urol Oncol 34: 215-220. [Crossref]

14. Wuttig D, Baier B, Fuessel S, Meinhardt M, Herr A, et al. (2009) Gene signatures of pulmonary metastases of renal cell carcinoma reflect the disease-free interval and the number of metastases per patient. Int J Cancer 125: 474-482. [Crossref]

Copyright: (C2018 Elfadaly A. This is an open-access article distributed under the terms of the Creative Commons Attribution License, which permits unrestricted use, distribution, and reproduction in any medium, provided the original author and source are credited. 